\title{
POSITIVE VALUES OF INHOMOGENEOUS 5-ARY QUADRATIC FORMS OF TYPE $(3,2)$
}

\author{
R. J. HANS-GILL AND MADHU RAKA
}

(Received 15 June 1979)

Communicated by A. J. van der Poorten

\begin{abstract}
Let $Q(x, y, z, t, u)$ be a real indefinite 5 -ary quadratic form of type $(3,2)$ and determinant $D(>0)$. Then given any real numbers $x_{0}, y_{0}, z_{0}, t_{0}, u_{0}$ there exist integers $x, y, z, t, u$ such that

$$
0<Q\left(x+x_{0}, y+y_{0}, z+z_{0}, t+t_{0}, u+u_{0}\right) \leqslant(16 D)^{1 / 5} .
$$
\end{abstract}

All the critical forms are also determined.

1980 Mathematics subject classification (Amer. Math. Soc.) : 10 E 20.

\section{Introduction}

Let $Q\left(x_{1}, x_{2}, \ldots, x_{n}\right)$ be a real indefinite quadratic form in $n$ variables with signature $(r, n-r), 0<r<n$ and determinant $D \neq 0$. It is known (see Blaney (1948)) that there exists a real number $\kappa$, depending only on $n$ and $r$, such that given any real numbers $c_{1}, c_{2}, \ldots, c_{n}$ the inequality

$$
0<Q\left(x_{1}+c_{1}, x_{2}+c_{2}, \ldots, x_{n}+c_{n}\right) \leqslant(\kappa|D|)^{1 / n}
$$

has a solution in integers $x_{1}, x_{2}, \ldots, x_{n}$. Let $\Gamma_{r, n-r}$ denote the infimum of all such constants $\kappa$. Davenport and Heilbronn (1947) proved that $\Gamma_{1,1}=4 . \Gamma_{2,1}=4$ was proved by Barnes (1961) and $\Gamma_{1,2}=8$ was obtained by Dumir (1967). Dumir (1968a, b) has also shown that $\Gamma_{3,1}=\frac{16}{3}$ and $\Gamma_{2,2}=16$. In this paper we shall prove that $\Gamma_{3,2}=16$. All the critical forms are also obtained. In a later paper we shall prove that $\Gamma_{4,1}=8$. More precisely here we prove :

THEOREM. Let $Q(x, y, z, t, u)$ be a real indefinite 5-ary quadratic form of type $(3,2)$ and determinant $D(>0)$. Then given any real numbers $x_{0}, y_{0}, z_{0}, t_{0}, u_{0}$ there exist integers $x, y, z, t, u$ such that 


$$
0<Q\left(x+x_{0}, y+y_{0}, z+z_{0}, t+t_{0}, u+u_{0}\right) \leqslant(16 D)^{1 / 5} .
$$

The sign of equality in (1.1) is necessary if and only if either

$$
Q(x, y, z, t, u) \sim \rho Q_{1}=\rho\left(x^{2}+y z+t u\right)
$$

or

$$
Q(x, y, z, t, u) \sim \rho Q_{2}=\rho\left(x^{2}+y^{2}-2 z^{2}-2 t u\right),
$$

where $\rho>0$. For $Q_{1}$, the sign of equality in (1.1) is necessary if and only if $\left(x_{0}, y_{0}, z_{0}, t_{0}, u_{0}\right) \equiv(0,0,0,0,0)(\bmod 1)$ while for $Q_{2}$ it is so if and only if $\left(x_{0}, y_{0}, z_{0}, t_{0}, u_{0}\right) \equiv\left(\frac{1}{2}, \frac{1}{2}, \frac{1}{2}, 0,0\right)(\bmod 1)$.

\section{Some lemmas}

In the course of the proof we shall use the following lemmas:

LEMMA 1. If $Q$ is as in the theorem, there exist integers $x_{1}, y_{1}, z_{1}, t_{1}, u_{1}$ such that

$$
0<Q\left(x_{1}, y_{1}, z_{1}, t_{1}, u_{1}\right) \leqslant(16 D)^{1 / 5} .
$$

The sign of equality in (2.1) is necessary if and only if $Q \sim \rho Q_{1}, \rho>0$.

This follows from some results of Watson $(1958,1968)$, Jackson (1969) and Oppenheim (1953a).

Let $\varphi(y, z, t, u)$ be a real indefinite quaternary quadratic form of type $(2,2)$ and determinant $D(>0)$. We shall need the following results :

Lemma 2. Given any real numbers $y_{0}, z_{0}, t_{0}, u_{0}$, there exist

$$
(y, z, t, u) \equiv\left(y_{0}, z_{0}, t_{0}, u_{0}\right)(\bmod 1)
$$

such that

$$
|\varphi(y, z, t, u)| \leqslant\left(\frac{1}{4} D\right)^{1 / 4}
$$

This is a theorem due to Birch (1958).

Lemma 3. There exist integers $y_{2}, z_{2}, t_{2}, u_{2}$ such that

$$
0<\varphi\left(y_{2}, z_{2}, t_{2}, u_{2}\right) \leqslant\left(\frac{81}{16} D\right)^{1 / 4}
$$

except when $\varphi(y, z, t, u) \sim \rho(y z+t u), \rho>0$. 
This is a theorem of Oppenheim (1953b).

LeMmA 4. There exist $(y, z, t, u) \equiv\left(y_{0}, z_{0}, t_{0}, u_{0}\right)(\bmod 1)$ such that

$$
0<\varphi(y, z, t, u) \leqslant(16 D)^{1 / 4} \text {. }
$$

This is a theorem of Dumir (1968b).

Lemma 5. Let $\psi(z, t, u)$ be an indefinite ternary quadratic form of type $(1,2)$ and determinant $D(>0)$. Then given any real numbers $z_{0}, t_{0}, u_{0}$ there exist $(z, t, u) \equiv\left(z_{0}, t_{0}, u_{0}\right)(\bmod 1)$ such that

$$
|\psi(z, t, u)| \leqslant\left(\frac{27}{100} D\right)^{1 / 3} .
$$

This is a theorem of Davenport (1948).

LeMMA 6. Let $\psi(z, t, u)$ be as in Lemma 5 . Then given any real $z_{0}, t_{0}, u_{0}$ there exist $(z, t, u) \equiv\left(z_{0}, t_{0}, u_{0}\right)(\bmod 1)$ such that

$$
-(D / 16)^{1 / 3} \leqslant \psi(z, t, u)<3 .(D / 16)^{1 / 3} .
$$

The sign of equality in (2.6) is necessary if and only if $\psi \sim \rho \psi_{1}$ or $\rho \psi_{2}, p>0$; where $\psi_{1}=-\left(z^{2}+t u\right), \psi_{2}=-2 z^{2}-t^{2}+u^{2}$. For $\psi_{1}$, the sign of equality in (2.6) is necessary if and only if $\left(z_{0}, t_{0}, u_{0}\right) \equiv\left(\frac{1}{2}, 0,0\right)(\bmod 1)$, while for $\psi_{2}$ it is necessary if and only if $\left(z_{0}, t_{0}, u_{0}\right) \equiv\left(\frac{1}{2}, \frac{1}{2}, \frac{1}{2}\right)(\bmod 1)$.

This follows from the theorem of Dumir (1969).

LEMMA 7. Let $\alpha, \beta, d$, be a real numbers with $d \geqslant 1$. Then given any real number $x_{0}$, there exists $x \equiv x_{0}(\bmod 1)$ such that

$$
0<(x+\alpha)^{2}-\beta^{2} \leqslant d
$$

provided

$$
\beta^{2} \begin{cases}\leqslant\left(\frac{d-1}{2}\right)^{2} & \text { if } d \text { is an integer, } \\ <\left(\frac{[d]}{2}\right)^{2} & \text { if } d \text { is not an integer. }\end{cases}
$$

Further strict inequality in (2.8) implies strict inequality in (2.7).

This is Lemma 6 of Dumir (1968a). 
LeMMA 8. Let $\alpha, \beta, d$, be as above. Then given any real $y_{0}$, there exists $y \equiv y_{0}(\bmod 1)$ such that

$$
0 \leqslant(y+\alpha)^{2}-\beta^{2}<d
$$

provided

$$
\beta^{2} \leqslant \begin{cases}\left(\frac{d-1}{2}\right)^{2} & \text { if } d \text { is an integer, } \\ \left(\frac{[d]}{2}\right)^{2} & \text { if } d \text { is not an integer. }\end{cases}
$$

Further strict inequality in (2.10) implies strict inequality in (2.9).

This lemma is a simple modification of Lemma 7 stated above, so we omit the proof.

LEMMA 9. Let $n$ be an integer $\geqslant 1$. Iffor $d>n, f(d)$ is an increasing function of $d$ and if

$$
f(d)<\left(\frac{d-1}{2}\right)^{2} \text { for } d \geqslant n+1,
$$

then for $n<d<n+1$, we have

$$
f(d)<\left(\frac{[d]}{2}\right)^{2} .
$$

This obvious result is useful in many calculations.

\section{Proof of the Theorem}

Let

By Lemma 1,

$$
m=\inf _{\substack{x, y, z, t, u \\ Q(x, y, z, t, u)>0}} Q(x, y, z, t, u)
$$

$$
0 \leqslant m \leqslant(16 D)^{1 / 5}
$$

If $m=0$, the result follows from a result of Watson (1960). So we can suppose $m>0$. Let $0<\varepsilon_{0}<\frac{1}{16}$ be a sufficiently small number. Then we can find integers $x_{1}, y_{1}, z_{1}, t_{1}, u_{1}$ such that 


$$
Q\left(x_{1}, y_{1}, z_{1}, t_{1}, u_{1}\right)=\frac{m}{1-\varepsilon} \leqslant(16 D)^{1 / 5}
$$

where $0 \leqslant \varepsilon<\varepsilon_{0}$. Since $\varepsilon<\frac{1}{16}$, we have g.c.d. $\left(x_{1}, y_{1}, z_{1}, t_{1}, u_{1}\right)=1$. By a suitable unimodular transformation we can suppose that

$$
Q(1,0,0,0,0)=\frac{m}{1-\varepsilon}
$$

and write

$$
Q(x, y, z, t, u)=\frac{m}{1-\varepsilon}\left\{\left(x+h y+g z+h^{\prime} t+g^{\prime} u\right)^{2}+\varphi(y, z, t, u)\right\}
$$

where

$$
|h| \leqslant \frac{1}{2}, \quad|g| \leqslant \frac{1}{2}, \quad\left|h^{\prime}\right| \leqslant \frac{1}{2}, \quad\left|g^{\prime}\right| \leqslant \frac{1}{2}
$$

and where $\varphi(y, z, t, u)$ is a real indefinite quadratic form of type $(2,2)$ with determinant

$$
D /\left(\frac{m}{1-\varepsilon}\right)^{5} \geqslant \frac{1}{16} \text {. }
$$

Equality in (3.2) occurs if and only if $Q \sim \rho Q_{1}$ (by Lemma 1). Also by the definition of $m$, for any integers $x, y, z, t, u$, we must have either $Q(x, y, z, t, u) \leqslant 0$ or $Q(x, y, z, t, u) \geqslant m$.

Because of homogeneity it suffices to prove

THEOREM A. Let $Q(x, y, z, t, u)=\left(x+h y+g z+h^{\prime} t+g^{\prime} u\right)^{2}+\varphi(y, z, t, u)$ where $\varphi(y, z, t, u)$ is a real indefinite quaternary quadratic form of type $(2,2)$ and determinant $D$ such that

$$
D \geqslant \frac{1}{16}, \quad\left(D=\frac{1}{16} \text { if and only if } Q \sim Q_{1}\right)
$$

and

$$
|h| \leqslant \frac{1}{2}, \quad|g| \leqslant \frac{1}{2}, \quad\left|h^{\prime}\right| \leqslant \frac{1}{2}, \quad\left|g^{\prime}\right| \leqslant \frac{1}{2}
$$

Suppose further that for integers $x, y, z, t, u$ we have either

$$
Q(x, y, z, t, u) \leqslant 0 \quad \text { or } \quad Q(x, y, z, t, u) \geqslant 1-\varepsilon
$$

where $0 \leqslant \varepsilon<\frac{1}{16}$ is sufficiently small.

Let

$$
d=(16 D)^{1 / 5} \text {. }
$$

Then given any real $x_{0}, y_{0}, z_{0}, t_{0}, u_{0}$, we can find 


$$
(x, y, z, t, u) \equiv\left(x_{0}, y_{0}, z_{0}, t_{0}, u_{0}\right)(\bmod 1)
$$

satisfying

$$
0<Q(x, y, z, t, u) \leqslant d .
$$

The sign of equality in (3.7) is necessary if and only if $Q \sim Q_{1}$ or $Q_{2}$.

\subsection{Proof of Theorem A.}

Lemma 10. If $Q(x, y, z, t, u)$ is as defined in Theorem $\mathrm{A}$, then for integers $y, z, t, u$ we have either

$$
\varphi(y, z, t, u) \leqslant 0 \quad \text { or } \quad \varphi(y, z, t, u) \geqslant \frac{3}{4}-\varepsilon .
$$

This result is similar to Lemma 4.1 of Dumir (1969), so we omit the proof.

LEMMA 11. If $Q=Q_{1}$, then (3.7) holds with strict inequality unless $\left(x_{0}, y_{0}, z_{0}, t_{0}, u_{0}\right) \equiv(0,0,0,0,0)(\bmod 1)$.

ProOF. Here $D=\frac{1}{16}$, so that $d=1$.

Case (i) $\left(y_{0}, z_{0}, t_{0}, u_{0}\right) \neq(0,0,0, \theta)(\bmod 1)$.

Without loss of generality we can suppose that $t_{0} \neq 0(\bmod 1)$. Choose $(x, y, z) \equiv\left(x_{0}, y_{0}, z_{0}\right)(\bmod 1)$ arbitrarily, $t \equiv t_{0}(\bmod 1)$ such that $0<|t| \leqslant \frac{1}{2}$ and then choose $u \equiv u_{0}(\bmod 1)$ to satisfy

$$
0<x^{2}+y z+t u \leqslant|t| \leqslant \frac{1}{2}<d .
$$

Case (ii) $\left(y_{0}, z_{0}, t_{0}, u_{0}\right) \equiv(0,0,0,0)(\bmod 1)$.

Take $y=z=t=u=0$ and choose $x \equiv x_{0}(\bmod 1)$ such that $0<x \leqslant 1$, so that

$$
0<x^{2}+y z+t u=x^{2} \leqslant 1=d .
$$

Strict inequality holds if $x_{0} \neq 0(\bmod 1)$. If $x_{0} \equiv 0(\bmod 1)$, then the sign of equality is necessary because $x^{2}+y z+t u$ takes only integral values.

So we can now suppose that $Q \nsucc Q_{1}$. By (3.3) $d \geqslant 1$, and $d=1$ if and only if $Q \sim Q_{1}$. Thus we have $d>1$ in the rest of the paper.

LEMMA 12. Let $v_{1}=d-\frac{1}{4}$ and $v_{2}>0$ be a real number satisfying 


$$
v_{2} \begin{cases}\leqslant\left(\frac{d-1}{2}\right)^{2} & \text { if } d \text { is an integer, } \\ <\left(\frac{[d]}{2}\right)^{2} & \text { if } d \text { is not an integer. }\end{cases}
$$

Suppose that we can find $(y, z, t, u) \equiv\left(y_{0}, z_{0}, t_{0}, u_{0}\right)(\bmod 1)$ such that

$$
-v_{2} \leqslant \varphi(y, z, t, u)<v_{1}
$$

then for any $x_{0}$, there exists $x \equiv x_{0}(\bmod 1)$ satisfying $(3.7)$. Further strict inequality in (3.10) implies strict inequality in (3.7).

ProOF. If $0<\varphi(y, z, t, u)<v_{1}$, choose $x \equiv x_{0}(\bmod 1)$ such that

$$
\left|x+h y+g z+h^{\prime} t+g^{\prime} u\right| \leqslant \frac{1}{2},
$$

so that

$$
0<Q(x, y, z, t, u)<\frac{1}{4}+v_{1}=d
$$

If $-v_{2} \leqslant \varphi(y, z, t, u) \leqslant 0$, then the result follows from Lemma 7 with $\alpha=h y+g z+h^{\prime} t+g^{\prime} u$ and $\beta^{2}=-\varphi(y, z, t, u)$.

LEMMA 13. If $d>8$, then (3.7) is true with strict inequality.

Proof. By Lemma 4 applied to $-\varphi(y, z, t, u)$, there exist $(y, z, t, u) \equiv$ $\left(y_{0}, z_{0}, t_{0}, u_{0}\right)(\bmod 1)$ such that

$$
0<-\varphi(y, z, t, u) \leqslant(16 D)^{1 / 4}
$$

i.e. $-d^{5 / 4}=-(16 D)^{1 / 4} \leqslant \varphi(y, z, t, u)<0$. The result will follow from Lemma 12 if we have

$$
d^{5 / 4}< \begin{cases}\left(\frac{d-1}{2}\right)^{2} & \text { if } d \geqslant 9 \\ \left(\frac{[d]}{2}\right)^{2} & \text { if } 8<d<9 .\end{cases}
$$

$f(d)=d^{5 / 4}$ is an increasing function for $d>1$. By Lemma 9 it is enough to verify the inequality for $d \geqslant 9$, which can be easily done.

LEMMA 14. If $3<d \leqslant 8$, then again (3.7) is true with strict inequality.

Proof. By Lemma 2, there exist $(y, z, t, u) \equiv\left(y_{0}, z_{0}, t_{0}, u_{0}\right)(\bmod 1)$ such that 


$$
|\varphi(y, z, t, u)| \leqslant\left(\frac{1}{4} D\right)^{1 / 4}=\left(\frac{d^{5}}{64}\right)^{1 / 4} .
$$

The result will follow from Lemma 12, if we have

$$
\left(\frac{d^{5}}{64}\right)^{1 / 4}<d-\frac{1}{4}
$$

and

$$
\left(\frac{d^{5}}{64}\right)^{1 / 4}< \begin{cases}\left(\frac{d-1}{2}\right)^{2} & \text { if } 4 \leqslant d \leqslant 8 \\ \left(\frac{[d]}{2}\right)^{2} & \text { if } 3<d<4 .\end{cases}
$$

We observe that by Lemma 9 , it is enough to verify ( 3.12$)$ for $4 \leqslant d \leqslant 8$. Verifications of these inequalities are easy and are left to the reader.

REMARK. For $1<d \leqslant 3$, we shall repeat the procedure of reduction described in Section 3. We use Lemma 3 on the homogeneous minimum of positive values of quaternary forms of type (2.2). So we first dispose of the exceptional forms.

LEMMA 15. If $\varphi(y, z, t, u) \sim \rho(y z+t u), \rho>0$ and $1<d \leqslant 3$, then (3.7) is true with strict inequality.

PROOF. Without loss of generality we can suppose that $\varphi(y, z, t, u)=\rho(y z+t u)$. So

$$
Q(x, y, z, t, u)=\left(x+h y+g z+h^{\prime} t+g^{\prime} u\right)^{2}+\rho(y z+t u) .
$$

By (3.4), $0 \leqslant Q(0,1,0,0,0)=h^{2} \leqslant \frac{1}{4}<1-\varepsilon$. Therefore (3.5) implies $h=0$. Similarly $g=g^{\prime}=h^{\prime}=0$. Therefore $Q(x, y, z, t, u)=x^{2}+\rho(y z+t u)$ and $D=\rho^{4} / 16$. Here $\rho / 2=\frac{1}{2}(16 D)^{1 / 4}=\frac{1}{2} d^{5 / 4}<d$, for $d \leqslant 3$. Now one can easily verify that $(3.7)$ is satisfied with strict inequality (proof is similar to that of Lemma 11).

\subsection{Proof of Theorem A continued}

From now on we can suppose that $1<d \leqslant 3$ and $\varphi(y, z, t, u) \psi \rho(y z+t u), \rho>0$. By Lemmas 3 and 10 , there exist integers $y_{2}, z_{2}, t_{2}, u_{2}$ with g.c.d. $\left(y_{2}, z_{2}, t_{2}, u_{2}\right)=1$ such that

$$
\frac{3}{4}-\varepsilon \leqslant a=\varphi\left(y_{2}, z_{2}, t_{2}, u_{2}\right) \leqslant\left(\frac{81}{16} D\right)^{1 / 4}=\frac{3}{4} d^{5 / 4} .
$$

By a suitable unimodular transformation we can suppose 


$$
\varphi(1,0,0,0)=a .
$$

So we can write

$$
\varphi(y, z, t, u)=a\left\{\left(y+f z+f^{\prime} t+f^{\prime \prime} u\right)^{2}+\psi(z, t, u)\right\}
$$

where

$$
\frac{3}{4}-\varepsilon \leqslant a \leqslant \frac{3}{4} d^{5 / 4}
$$

and $\psi(z, t, u)$ is a real indefinite ternary quadratic form of type $(1,2)$ and determinant $D / a^{4}$. In view of Lemma 12 , it is enough to prove that there exist $(y, z, t, u) \equiv\left(y_{0}, z_{0}, t_{0}, u_{0}\right)(\bmod 1)$ such that

$$
-\frac{v}{a} \leqslant\left(y+f z+f^{\prime} t+f^{\prime \prime} u\right)^{2}+\psi(z, t, u)<\frac{4 d-1}{4 a}
$$

and that strict inequality holds if $d$ is not an integer; where

$$
v= \begin{cases}1 & \text { if } 2<d \leqslant 3 \\ \frac{1}{4} & \text { if } 1<d \leqslant 2\end{cases}
$$

Let

$$
\mu_{1}=\frac{4 d-1-a}{4 a} \text { and } \lambda=\frac{4 d-1}{4 a}+\frac{v}{a} .
$$

Using (3.13) one can easily verify that $\mu_{1}>0$ and $\lambda>1$. The proof of the following lemma is similar to that of Lemma 12 and is omitted. (Here we use Lemma 8 instead of Lemma 7.)

LEMMA 16. Let

$$
0<\mu_{2} \leqslant \begin{cases}\left(\frac{\lambda-1}{2}\right)^{2}+\frac{v}{a} & \text { if } \lambda \text { is an integer } \\ \left(\frac{[\lambda]}{2}\right)^{2}+\frac{v}{a} & \text { if } \lambda \text { is not an integer. }\end{cases}
$$

Suppose that we can find $(z, t, u) \equiv\left(z_{0}, t_{0}, u_{0}\right)(\bmod 1)$ such that

$$
-\mu_{2} \leqslant \psi(z, t, u)<\mu_{1}
$$

and strict inequality holds in (3.17) if $d$ is not an integer. Then there exists $y \equiv y_{0}$ (mod 1) such that (3.14) holds. Further strict inequality in (3.17) implies strict inequality in (3.14).

LEMMA 17. If $2<d \leqslant 3$, then (3.17) and hence (3.14) is true with strict inequality. 
ProOF. In this case $v=1$, so that $\lambda=(3+4 d) / 4 a$. By Lemma 5 , we can find $(z, t, u) \equiv\left(z_{0}, t_{0}, u_{0}\right)(\bmod 1)$ such that

$$
|\psi(z, t, u)| \leqslant\left(\frac{27}{100} \frac{D}{a^{4}}\right)^{1 / 3}=\left(\frac{27 d^{5}}{1600 a^{4}}\right)^{1 / 3} .
$$

Then (3.17) will hold with strict inequality if we have

$$
\left(\frac{27 d^{5}}{1600 a^{4}}\right)^{1 / 3}<\frac{4 d-1-a}{4 a}
$$

and

$$
\left(\frac{27 d^{5}}{1600 a^{4}}\right)< \begin{cases}\left(\frac{\lambda-1}{2}\right)^{2}+\frac{1}{a} & \text { if } \lambda \text { is an integer } \\ \left(\frac{[\lambda]}{2}\right)^{2}+\frac{1}{a} & \text { if } \lambda \text { is not an integer. }\end{cases}
$$

Verification of (3.18) is straightforward. So we proceed to verify (3.19). Let

$$
n<\lambda=\frac{3+4 d}{4 a} \leqslant n+1, \quad n=1,2,3, \ldots
$$

Then (3.19) will be satisfied if we have

$$
\left(\frac{27 d^{5}}{1600 a^{4}}\right)^{1 / 3}<\frac{n^{2}}{4}+\frac{1}{a} \text { for all } n \geqslant 1 .
$$

That is,

$$
\frac{27 d^{5}}{1600 a^{4}}<a^{4}\left(\frac{n^{2}}{4}+\frac{1}{a}\right)^{3}=g(a) \quad \text { (say). }
$$

$g(a)$ is an increasing function of $a$ and $a \geqslant(3+4 d) / 4(n+1)$, therefore

$$
g(a) \geqslant g\left(\frac{3+4 d}{4(n+1)}\right)=\frac{(3+4 d)\left\{n^{2}(3+4 d)+16(n+1)\right\}^{3}}{4^{7}(n+1)^{4}} .
$$

So we shall have $(3.20)$ if

$$
\frac{(3+4 d)\left\{n^{2}(3+4 d)+16(n+1)\right\}^{3}}{(n+1)^{4} d^{5}}>4^{4} \cdot \frac{27}{25}
$$

As the left-hand side of (3.21) is clearly a decreasing function of $d$ and $d \leqslant 3$, one can easily check that (3.21) is true for all $n \geqslant 1$. This proves (3.20) and hence (3.19).

LEMMA 18. If $1<d \leqslant 2$, then again (3.17) and hence (3.14) is true. Moreover (3.14) holds with strict inequality unless $d=2, a=1$ and $\psi, y_{0}, z_{0}, t_{0}$ are such that equality is necessary in (2.6). 
ProOf. In this case $v=\frac{1}{4}$, so that $\lambda=d / a$. By (3.13),

$$
\lambda=\frac{d}{a} \leqslant \frac{2}{3 / 4-\varepsilon}<3,
$$

for sufficiently small $\varepsilon$. We distinguish two cases :

Case (i) $2<\lambda<3$.

In this case

$$
\left(\frac{[\lambda]}{2}\right)^{2}+\frac{v}{a}=1+\frac{1}{4 a}=\frac{1+4 a}{4 a} .
$$

So we have to prove that there exist $(z, t, u) \equiv\left(z_{0}, t_{0}, u_{0}\right)(\bmod 1)$ such that

$$
-\frac{1+4 a}{4 a}<\psi(z, t, u)<\frac{4 d-1-a}{4 a} .
$$

By Lemma 5 , we can find $(z, t, u) \equiv\left(z_{0}, t_{0}, u_{0}\right)(\bmod 1)$ such that

$$
|\psi(z, t, u)| \leqslant\left(\frac{27 d^{5}}{1600 a^{4}}\right)^{1 / 3}
$$

Therefore (3.22) follows from (3.23) if we have

$$
\left(\frac{27 d^{5}}{1600 a^{4}}\right)^{1 / 3}<\min \left(\frac{1+4 a}{4 a}, \frac{4 d-1-a}{4 a}\right)
$$

This inequality can be easily checked.

Case (ii) $1<\lambda \leqslant 2$.

In this case we have to prove that there exist $(z, t, u) \equiv\left(z_{0}, t_{0}, u_{0}\right)(\bmod 1)$ such that

$$
-\frac{1+a}{4 a} \leqslant \psi(z, t, u)<\frac{4 d-a-1}{4 a}
$$

and strict inequality holds if $d$ is not an integer. By Lemma 6, there exist $(z, t, u) \equiv\left(z_{0}, t_{0}, u_{0}\right)(\bmod 1)$ such that

$$
-\left(\frac{D}{16 a^{4}}\right)^{1 / 3} \leqslant \psi(z, t, u)<3\left(\frac{D}{16 a^{4}}\right)^{1 / 3} .
$$

Therefore (3.24) will hold if we have

$$
3\left(\frac{D}{16 a^{4}}\right)^{1 / 3}=3\left(\frac{d^{5}}{256 a^{4}}\right)^{1 / 3} \leqslant \frac{4 d-1-a}{4 a}
$$


and

$$
\left(\frac{d^{5}}{256 a^{4}}\right)^{1 / 3} \leqslant \frac{1+a}{4 a}
$$

with strict inequality if $d \neq 2$.

Now

$$
\frac{4 d-1-a}{12 a} \leqslant \frac{1+a}{4 a} \text { for } a \geqslant \frac{d}{2} \text { and } d \leqslant 2
$$

Equality holds if and only if $d=2, a=\frac{1}{2} d=1$. Therefore it is enough to prove that (3.25) holds and equality is necessary only for $d=2$. We shall have (3.25) if and only if

$$
\frac{27}{256} d^{5} \leqslant a\left(d-\frac{1-a}{4}\right)^{3}=g(a) \quad(\text { say })
$$

$g(a)$ increases or decreases according as $a<d-\frac{1}{4}$ or $a>d-\frac{1}{4}$ and since $\frac{1}{2} d<d-\frac{1}{4}$, (3.27) will be true for $d / 2 \leqslant a<\frac{3}{4} d^{5 / 4}$ if

$$
\min \left(g\left(\frac{d}{2}\right), g\left(\frac{3}{4} d^{5 / 4}\right)\right) \geqslant \frac{27}{256} d^{2}
$$

Now

$$
g\left(\frac{d}{2}\right)=\frac{d(7 d-2)^{3}}{2.8^{3}} \geqslant \frac{27}{256} d^{5}
$$

if

$$
f(d)=\frac{(7 d-2)^{3}}{d^{4}} \geqslant 3^{3} \cdot 4 .
$$

$f(d)$ increases for $d \leqslant \frac{8}{7}$ and decreases for $d \geqslant \frac{8}{7}$, therefore for $1<d \leqslant 2$,

$$
f(d) \geqslant \min (f(1), f(2))=f(2)=3^{3} \cdot 4,
$$

and strict inequality holds unless $d=2$. The inequality $g\left(\frac{3}{4} d^{5 / 4}\right)>\frac{27}{256} d^{5}$ can be easily verified.

Therefore (3.27) is satisfied with strict inequality unless $d=2, a=\frac{1}{2} d=1$. Hence (3.24) is satisfied. Equality holds in (3.24) only if $d=2, a=1$ and $\psi, z_{0}, t_{0}, u_{0}$ are such that the sign of equality is necessary in (2.6).

This completes the proof of the lemma. 


\section{The case of equality}

LEMMA 19. The sign of equality in (3.7) is necessary if and only if $Q \sim Q_{2}$. For $Q_{2}$ it is necessary if and only if

$$
\left(x_{0}, y_{0}, z_{0}, t_{0}, u_{0}\right) \equiv\left(\frac{1}{2}, \frac{1}{2}, \frac{1}{2}, 0,0\right) \quad(\bmod 1) .
$$

ProOF. Equality can be necessary in (3.7) only if it is necessary (3.14). This happens only if $d=2, a=1$ and $\psi, z_{0}, t_{0}, u_{0}$ are such that equality is necessary in (2.6) (see Lemma 18). Thus we must have $\psi \sim \rho \psi_{1}$ or $\rho \psi_{2}, \rho>0$. For $\psi_{1}$ we must have $\left(z_{0}, t_{0}, u_{0}\right) \equiv\left(\frac{1}{2}, 0,0\right)$, while for $\psi_{2}$ we have $\left(z_{0}, t_{0}, u_{0}\right) \equiv\left(\frac{1}{2}, \frac{1}{2}, \frac{1}{2}\right)(\bmod 1)$.

Case (i). $\psi_{1}(z, t, u)=-\rho\left(z^{2}+t u\right),\left(z_{0}, t_{0}, u_{0}\right) \equiv\left(\frac{1}{2}, 0,0\right)(\bmod 1)$. Then

$$
\frac{1}{4} \rho^{3}=\frac{D}{a^{4}}=\frac{d^{5}}{16 a^{4}}=2,
$$

so that $\rho=2$. Therefore

$$
\varphi(y, z, t, u)=\left(y+f z+f^{\prime} t+f^{\prime \prime} u\right)^{2}-2 z^{2}-2 t u .
$$

By a suitable unimodular transformation we can suppose that

$$
|f| \leqslant \frac{1}{2},\left|f^{\prime}\right| \leqslant \frac{1}{2},\left|f^{\prime \prime}\right| \leqslant \frac{1}{2}
$$

If $f^{\prime \prime} \neq 0$, then

$$
0<\varphi(0,0,0,1)=f^{\prime \prime 2} \leqslant \frac{1}{4}<\frac{3}{4}-\varepsilon .
$$

This contradicts (3.8) Therefore $f^{\prime \prime}=0$. Similarly, consideration of $\varphi(0,0,1,0)$ and $\varphi(0,1,-1,1)$ gives $f^{\prime}=f=0$. Hence $\varphi(y, z, t, u)=y^{2}-2 z^{2}-2 t u$. For equality to occur in (3.14), the inequality

$$
-\frac{1}{4}<\left(y+y_{0}\right)^{2}-2\left(z+\frac{1}{2}\right)^{2}-2 t u<d-\frac{1}{4}=\frac{7}{4}
$$

should have no solution in integers $y, z, t$ and $u$. Take $z=t=u=0$ and choose the integer $y$ such that $\left|y+y_{0}\right| \leqslant \frac{1}{2}$, then (4.2) is solvable unless $y_{0} \equiv \frac{1}{2}(\bmod 1)$. Therefore,

$$
\begin{aligned}
& Q(x, y, z, t, u)=\left(x+h y+g z+h^{\prime} t+g^{\prime} u\right)^{2}+y^{2}-2 z^{2}-2 t u \\
& \left(y_{0}, z_{0}, t_{0}, u_{0}\right) \equiv\left(\frac{1}{2}, \frac{1}{2}, 0,0\right) \quad(\bmod 1) .
\end{aligned}
$$

Considering $Q(0,0,0,0,1), Q(0,0,0,1,0)$ and $Q(0,0,1,-1,1)$ and using (3.4), (3.5) we get $g=h^{\prime}=g^{\prime}=0$. Therefore $Q(x, y, z, t, u)=(x+h y)^{2}+y^{2}-2 z^{2}-2 t u$. If equality is to be necessary in (3.7), the inequality 


$$
\begin{array}{r}
0<F(x, y, z, t, u)=\left(x+x_{0}+h\left(y+\frac{1}{2}\right)\right)^{2}+\left(y+\frac{1}{2}\right)^{2} \\
-2\left(z+\frac{1}{2}\right)^{2}-2 t u<d=2
\end{array}
$$

should have no solution in integers $x, y, z, t, u$. Now $0<F(x, 0,0,0,0)<2$ is solvable for integer $x$ unless $x_{0}+\frac{1}{2} h \equiv \frac{1}{2}(\bmod 1)$. Also $0<F(x,-1,0,0,0)<2$ is solvable in integer $x$ unless $x_{0}-\frac{1}{2} h \equiv \frac{1}{2}(\bmod 1)$. Thus $(4.3)$ is solvable unless $h \equiv 0(\bmod 1)$. Since $|h| \leqslant \frac{1}{2}$ from (3.4), we must have $h=0$ Then $x_{0} \equiv \frac{1}{2}(\bmod 1)$. Hence

$$
Q(x, y, z, t, u)=x^{2}+y^{2}-2 z^{2}-2 t u=Q_{2}
$$

and

$$
\left(x_{0}, y_{0}, z_{0}, t_{0}, u_{0}\right) \equiv\left(\frac{1}{2}, \frac{1}{2}, \frac{1}{2}, 0,0\right)(\bmod 1) .
$$

Considering congruence module 8 , one can see that the sign of equality is necessary in this case.

Case (ii). $\psi_{2}(z, t, u)=-\rho\left(2 z^{2}+t^{2}-u^{2}\right),\left(z_{0}, t_{0}, u_{0}\right) \equiv\left(\frac{1}{2}, \frac{1}{2}, \frac{1}{2}\right)(\bmod 1)$ Proceeding as above, one can see that equality is necessary in (3.7) if and only if

$$
Q=x^{2}+y^{2}-2 z^{2}-t^{2}+u^{2}
$$

and

$$
\left(x_{0}, y_{0}, z_{0}, t_{0}, u_{0}\right) \equiv\left(\frac{1}{2}, \frac{1}{2}, \frac{1}{2}, \frac{1}{2}, \frac{1}{2}\right)(\bmod 1) .
$$

Since

$$
x^{2}+y^{2}-2 z^{2}-t^{2}+u^{2}=(x-t-u)^{2}+y^{2}-2 z^{2}+2(x-t)(t+u),
$$

$Q \sim x^{2}+y^{2}-2 z^{2}+2 t u \sim Q_{2}$. Therefore this case does not give us a new form.

The proof of Theorem A follows from Lemmas 10 to 19 and thus our theorem is proved.

\section{Acknowledgements}

The authors are very grateful to Professor R. P. Bambah and Professor V. C. Dumir for many useful discussions during the preparation of this paper. The authors are also grateful to the referee for some useful comments.

\section{References}

E. S. Barnes (1961), 'The positive values of inhomogeneous ternary quadratic forms', J. Austral. Math. Soc. 2, 127-132. 
B. J. Birch (1958), 'The inhomogeneous minimum of quadratic forms of signature zero'. Acta Arith. 4, $85-98$.

H. Blaney (1948), 'Indefinite quadratic forms in $n$ variables', J. London Math. Soc. 23, 153-160.

H. Davenport (1948), 'Non-homogeneous ternary quadratic forms', Acta Math. 80, 65-95.

H. Davenport and H. Heilbronn (1947), 'Asymmetric inequalities for non-homogeneous linear forms,' $J$. London Math. Soc. 22, 53-61.

V. C. Dumir (1967), 'Asymmetric inequalities for non-homogeneous ternary quadratic forms.' Proc. Cambridge Philos. Soc. 63, 291-303.

V. C. Dumir (1968a), 'Positive values of inhomogeneous quaternary quadratic forms (I),' J. Austral. Math. Soc. 8, 87-101.

V. C. Dumir (1968b), 'Positive values of inhomogeneous quaternary quadratic forms (II),' J. Austral. Math. Soc. 8, 287-303.

V. C. Dumir (1969), 'Asymmetric inequality for non-homogeneous ternary quadratic forms', J. Number Theory 1, 326-345,

H. Jackson (1969), 'Small positive values of indefinite quadratic forms.' J. London Math. Soc. (2) 1, 643-659.

A. Oppenheim (1953a), 'Values of quadratic forms II', Quart. J. Math. Oxford Ser. 4, 60-66.

A. Oppenheim (1953b), 'One sided inequalities for quadratic forms (II) quaternary forms', Proc. London Math. Soc. 3, 417-429.

G. L. Watson (1958), 'One sided inequalities for integral quadratic forms,' Quart J. Math. Oxford Ser. 9, 99-108.

G. L. Watson (1960), 'Indefinite quadratic polynomials', Mathematika 7, 141-144.

G. L. Watson (1968), 'Asymmetric inequalities for indefinite quadratic forms,' Proc. London Math. Soc.(3) $18,95-113$.

Centre of Advanced Study in Mathematics

Panjab University

Chandigarh-160014

India 